\title{
Effective factors on advanced manufacturing technology implementation performance: a review
}

\begin{abstract}
This study reviews an extensive body of literature to investigate the factors effective on performance of companies implementing Advanced Manufacturing Technology (AMT). The purpose of this study is to provide a comprehensive viewpoint of issues related to successful AMT implementation and offer some directions to managers and investigators to make a company well-prepared to accept technology. The factors are grouped into three categories: technological, organizational and internal/external. The literature showed that in order to have a fruitful result from AMT investment, the organizational structure and culture, operational strategy and human resource should be organized and integrated appropriately with each other to avoid probable barriers and problems. Proposed framework can be used as a guideline for managers and investors in improving their AMT implementation process.
\end{abstract}

Keyword: Advanced manufacturing technology; Performance; Strategic framework; Technology fit 